\title{
Risk Factors for Refractory Diabetic Macular Oedema after Sub-Tenon's Capsule Triamcinolone Acetonide Injection
}

\author{
Toshiyuki Oshitari, Yuta Kitamura, Sakiko Nonomura, Miyuki Arai, \\ Yoko Takatsuna, Eiju Sato, Takayuki Baba, and Shuichi Yamamoto \\ Department of Ophthalmology and Visual Science, Chiba University Graduate School of Medicine, Inohana 1-8-1, \\ Chuo-ku, Chiba Prefecture, Chiba 260-8670, Japan \\ Correspondence should be addressed to Toshiyuki Oshitari; tarii@aol.com
}

Received 5 June 2015; Revised 5 September 2015; Accepted 6 September 2015

Academic Editor: Ciro Costagliola

Copyright (C) 2015 Toshiyuki Oshitari et al. This is an open access article distributed under the Creative Commons Attribution License, which permits unrestricted use, distribution, and reproduction in any medium, provided the original work is properly cited.

\begin{abstract}
The purpose of this study is to identify the risk factors for a recurrence or persistence of diabetic macular oedema (DME) after a sub-Tenon's capsule triamcinolone acetonide (STTA) injection. The medical records of 124 patients (124 eyes) treated by STTA were reviewed. The age, sex, HbAlc level, best-corrected visual acuity, central macular thickness, insulin use, pioglitazone use, systemic hypertension, serous retinal detachment, proteinuria, panretinal photocoagulation, microaneurysm photocoagulation (MAPC), subthreshold micropulse diode laser photocoagulation (SMDLP), cataract surgery, and history of vitrectomy were examined by logistic regression analysis. Procedures of MAPC and SMDLP were significantly associated with DME treated with STTA $(P=$ $0.0315, P=0.04$, resp.). However, a history of vitrectomy was found to have significantly fewer recurrences or persistent DME after STTA $(P=0.0464)$. In conclusion, patients who required combined MAPC or SMDLP with a STTA injection had significantly higher refractoriness to STTA, but postvitrectomy may prevent the recurrence or persistence of DME after STTA injection.
\end{abstract}

\section{Introduction}

Diabetic macular oedema (DME) is one of the main causes for reduced visual acuity in patients with diabetes [1]. A recent meta-analysis examined the prevalence of DME in 22,896 diabetic patients and found that $6.81 \%$ of diabetic patients had DME [2]. Several prospective, randomized studies showed that intravitreal injections of antivascular endothelial growth factor (VEGF) drugs were effective in reducing macular thickness and improving the visual acuity in patients with DME [3-6]. However, the injections had to be repeated which increased the risk of postintravitreal anti-VEGF endophthalmitis and the medical expenses. For example, in the pooled analysis of the RESOLVE and the RESTORE studies, the incidence of endophthalmitis was $1.4 \%$ at 1 year for multiple injections [3].

Growing evidence indicates an association between the intraocular inflammation induced by diabetic stress and the development and progression of DME [7]. Several basic studies demonstrated that steroids upregulate the tight junction proteins, occludin and ZO-1, tighten the retinal blood barrier [8], and reduce the expression of VEGF $[9,10]$. Thus, posterior sub-Tenon's capsule injection of triamcinolone acetonide (STTA) [11] and intravitreal injections of triamcinolone acetonide (IVTA) [12] have been used to treat DME.

IVTA has a higher risk of endophthalmitis and elevation of the intraocular pressure than STTA. The Japanese survey of triamcinolone acetonide for ocular diseases reported that the incidence of endophthalmitis by IVTA and STTA was $0.12 \%$ and $0.008 \%$, respectively, and that the incidence of glaucoma requiring filtration surgery after IVTA and STTA was $0.56 \%$ and $0.26 \%$, respectively [13]. Thus, STTA has a lower risk of endophthalmitis and secondary glaucoma than IVTA.

Our recent study indicated that the short-term effect of STTA for DME is comparable to that of pars plana vitrectomy [14]. However, the benefits of steroid therapies were no longer evident at 6 months [15]. Thus, repeated injections 
or additional treatments such as laser photocoagulation are usually required for the treatment of DME.

The main purpose of this study was to identify the risk factors that led to a recurrence or persistence of DME after a STTA injection.

\section{Methods}

The medical records of 124 eyes of 124 patients with DME that had STTA between January 2010 and July 2011 at the Chiba University Hospital were reviewed. All of the procedures conformed to the tenets of the World Medical Association Declaration of Helsinki. A signed informed consent was obtained from all patients regarding the procedures to be performed, and approval for this study was obtained from the Institutional Review Board of Chiba University Graduate School of Medicine.

The definition of a recurrence of DME in this study was an eye which initially had $\mathrm{a} \geqq 30 \%$ decrease of central macular thickness (CMT) compared with the baseline within 1 year after STTA but then increased by $\geqq 30 \%$. The definition of a persistence of DME was an eye which had $<30 \%$ decrease of the CMT within 1 year after STTA. Seventy-four patients (59.7\%; 42 men, 32 women) had a persistent DME or a recurrence within 1 year. In the eyes with a recurrence, the mean interval until the recurrence was $7.7 \pm 3.5$ months.

The possible risk factors for a recurrence or persistence of a DME after STTA were the age, sex, glycohemoglobin Alc (HbAlc) level, best-corrected visual acuity (BCVA), CMT, insulin use, pioglitazone use, systemic hypertension, serous retinal detachment (SRD), proteinuria, panretinal photocoagulation (PRP), microaneurysm photocoagulation (MAPC), subthreshold micropulse diode laser photocoagulation (SMDLP), cataract surgery, and history of vitrectomy. All possible risk factors for recurrence or persistence of DME after STTA were determined by logistic regression analysis. A $P<0.05$ was considered significant.

\section{Results}

The baselines clinical characteristics of the 124 patients with DME are shown in Table 1 . The DME of 50 eyes (40.3\%) was improved after the STTA injection without any additional treatments, $52(41.9 \%)$ had a recurrence of DME, and 22 eyes $(17.7 \%)$ had a persistence of the DME after STTA injection. All of the results of multiple logistic regression analyses of the risk factors for a recurrence or persistence of DME after the STTA injection are shown in Table 2.

At the time of treatment, the mean age was $60.3 \pm 13.1$ years, mean $\mathrm{HbAlc}$ was $6.7 \pm 1.2 \%$, mean BCVA was $0.6 \pm$ $0.4 \log$ MAR units, and mean CMT was $539.2 \pm 156.7 \mu \mathrm{m}$. Thirty-eight patients (30.6\%) used insulin, 10 used pioglitazone (8.1\%), 55 (44.4\%) had hypertension, and 47 (37.9\%) had proteinuria. Fifty-five patients $(44.4 \%)$ underwent PRP and 25 patients $(20.2 \%)$ underwent cataract surgery. None of these factors was found to be significant risk factors (Tables 1 and 2).
TABLE 1: Baseline clinical characteristics of 124 patients with DME.

\begin{tabular}{lc}
\hline Factors & Cases $(124$ eyes) \\
\hline Sex, men $:$ women $(n)$ & $71: 53$ \\
Mean age (y.o.) & $60.3 \pm 13.1$ \\
Mean HbA1c $(\%)$ & $6.7 \pm 1.2$ \\
Hypertension, $+:-(n)$ & $55: 69$ \\
Insulin use, $+:-(n)$ & $38: 86$ \\
Pioglitazone use, $+:-(n)$ & $10: 114$ \\
Proteinuria, $+:-(n)$ & $47: 77$ \\
Mean BCVA $(\operatorname{logMAR})$ & $0.6 \pm 0.4$ \\
Mean CMT $(\mu \mathrm{m})$ & $539.2 \pm 156.7$ \\
SRD, $+:-(n)$ & $33: 91$ \\
MAPC, $+:-(n)$ & $49: 75$ \\
SMDLP, $+:-(n)$ & $13: 111$ \\
PRP, $+:-(n)$ & $55: 69$ \\
Vitrectomy, $+:-(n)$ & $17: 107$ \\
Cataract surgery, $+:-(n)$ & $25: 99$ \\
\hline
\end{tabular}

BCVA: best-corrected visual acuity, CMT: central macular thickness, SRD: serous retinal detachment, MAPC: microaneurysm photocoagulation, SMDLP: subthreshold micropulse diode laser photocoagulation, and PRP: panretinal photocoagulation.

Forty-nine patients (39.5\%) underwent MAPC and 13 patients (10.5\%) underwent SMDLP combined with the STTA injection. These procedures were found to be significantly associated with a persistence or recurrence of the DME $(P=0.0315, P=0.04$, resp.; Table 2$)$. On the other hand, 17 patients (13.7\%) with a history of PPV had significantly fewer recurrences or persistence of DME after STTA injection $(P=$ 0.0464; Table 2).

\section{Discussion}

The results indicated that $40 \%$ of patients with DME were successfully treated with a single STTA injection without any additional treatments for at least 1 year. On the other hand, $60 \%$ of DME patients had a recurrence or persistence of DME after a single STTA injection and repeated STTA injections or other treatments were required.

The specific indications for MAPC and SMDLP were not determined but the patients with microaneurysms at the posterior pole underwent STTA combined with MAPC immediately or within one month after the STTA injection. The effect of the STTA injection for DME is rapid, and our results indicate that the CMT was significantly reduced 1 month after the STTA injection [14]. In our hospital, SMDLP was determined to be a better treatment than grid laser photocoagulation for DME [16]. Thus, the refractory DME patients without microaneurysms underwent SMDLP and not grid laser photocoagulation as additional treatment after STTA. Basically, both MAPC and SMDLP tended to be performed for patients with mild to moderate DME with CMT $>500 \mu \mathrm{m}$ in our hospital. Therefore, patients with severe DME with CMT $<500 \mu \mathrm{m}$ underwent STTA first, followed by undergoing MAPC or SMDLP. 
TABLE 2: Multiple logistic regression analysis of a recurrence and/or persistence of DME after STTA.

\begin{tabular}{|c|c|c|c|c|c|}
\hline Factors & Recurrence (+) & Recurrence (-) & Odds ratio & $95 \% \mathrm{CI}$ & $P$ value \\
\hline Sex, men : women $(n)$ & $42: 32$ & $29: 21$ & 0.718 & $0.034-0.973$ & 0.4846 \\
\hline Mean age (y.o.) & $61.6 \pm 13.0$ & $58.5 \pm 13.2$ & 1.012 & $0.978-1.049$ & 0.4883 \\
\hline Mean HbAlc (\%) & $6.83 \pm 1.32$ & $6.59 \pm 1.09$ & 1.239 & $0.833-1.843$ & 0.2906 \\
\hline Hypertension, + :- & $38: 36$ & $17: 33$ & 1.926 & $0.760-4.879$ & 0.1669 \\
\hline Insulin use, $+:-(n)$ & $24: 50$ & $14: 36$ & 1.367 & $0.525-3.562$ & 0.5218 \\
\hline Pioglitazone use, $+:-(n)$ & $8: 66$ & $2: 48$ & 2.416 & $0.373-15.628$ & 0.3544 \\
\hline Proteinuria, $+:-(n)$ & $30: 44$ & $17: 33$ & 1.103 & $0.420-2.894$ & 0.8422 \\
\hline Mean BCVA (logMAR) & $0.61 \pm 0.37$ & $0.52 \pm 0.33$ & 2.054 & $0.523-8.065$ & 0.3022 \\
\hline Mean CMT $(\mu \mathrm{m})$ & $557.2 \pm 143.7$ & $512.8 \pm 172.2$ & 1.002 & 0.999-1.005 & 0.1800 \\
\hline $\mathrm{SRD},+:-(n)$ & $22: 52$ & $11: 39$ & 1.012 & $0.370-2.769$ & 0.9821 \\
\hline MAPC, $+:-(n)$ & $35: 39$ & $14: 36$ & 2.566 & $1.087-6.058$ & 0.0315 \\
\hline SMDLP, $+:-(n)$ & $11: 63$ & $2: 48$ & 7.772 & $1.098-55.004$ & 0.0400 \\
\hline $\mathrm{PRP},+:-(n)$ & $33: 41$ & $22: 28$ & 0.951 & $0.390-2.321$ & 0.9124 \\
\hline Vitrectomy, $+:-(n)$ & $7: 67$ & $10: 40$ & 0.182 & $0.034-0.973$ & 0.0464 \\
\hline Cataract surgery, $+:-(n)$ & $15: 59$ & $10: 40$ & 1.355 & $0.336-5.460$ & 0.6691 \\
\hline
\end{tabular}

BCVA: best-corrected visual acuity, CMT: central macular thickness, SRD: serous retinal detachment, MAPC: microaneurysm photocoagulation, SMDLP: subthreshold micropulse diode laser photocoagulation, and PRP: panretinal photocoagulation.

The results of the logistic regression analysis indicated that both MAPC and SMDLP were risk factors for a recurrence or persistence of DME because these patients tended to have refractory DME and needed to undergo additional treatments. Although patients who underwent MAPC had an enough population size, smaller number of patients underwent SMDLP compared to MAPC. Thus, the result of the logistic regression analysis for SMDLP should be interpreted with caution.

Recently Ribeiro et al. suggested that a high microaneurysm turnover rate (sum of the microaneurysm formation and disappearance rates) was a higher risk for developing clinical significant macular oedema (CSME) over a 2year period [17]. Haritoglou et al. demonstrated that high microaneurysm formation rate was a predictive marker for progression to CSME for a period of up to 5 years [18]. Taken together, microaneurysm formation is probably a sign of severe diabetic stress including oxidative stress in the macula of diabetic patients, and the requirement of MAPC combined with STTA injections may be necessary to treat the DME. However, these patients may increase diabetic stress including oxidative stress in the macula; further pathological changes such as Müller cell swelling, retinal pigment epithelium (RPE) dysfunction, or blood-retinal barrier dysfunction may be accompanied. Such pathological changes may cause refractoriness of DME after STTA with MAPC.

Although the precise mechanism of the effect of SMDLP is unclear, SMDLP may stimulate and activate RPE and improve to draw out the excessive fluid in the retina. But steroid can affect the function of RPE in diabetic patients. Thus, STTA may not be fitted with SMDLP because of exacerbating RPE function.

Vitrectomized eyes had a significantly lower risk for recurrence or persistence of DME after the STTA injection. One possible reason for this is that vitrectomized eyes have no vitreomacular traction. Another possible reason is that, in vitrectomized eyes, pathological cytokines, such as VEGF or IL-6, can easily diffuse and are not in contact with the macula for a long period. Thus, a STTA injection may be one of the options for treatment of DME developing in vitrectomized eyes but a careful management of the steroid response is needed because glaucoma infiltration surgeries are difficult to perform in vitrectomized eyes.

The HbAlc level is known to be a major risk factor for developing DME $[19,20]$. However, in this study, the HbAlc level was not found to be a significant risk factor for recurrence or persistence of DME after a STTA injection from the logistic regression analysis. We have classified the grades of DM control as good control group ( $\mathrm{HbAlc}<$ $6.5 \%)$, moderated control group $(6.5 \% \leqq \mathrm{HbAlc} \leqq 8.0 \%)$, and poor control group $(8.0 \%<\mathrm{HbAlc})$ and reevaluated whether the DM control was risk factors for recurrence or persistence of DME after STTA injection. But the DM controls have not been identified as risk factors for recurrence or persistence of DME after STTA injection $(P=0.2203)$. However, $80 \%$ of the patients with HbAlc levels $>9.0 \%$ had a recurrence or persistence of the DME after the STTA injection. Thus, a poor glycemic control seems to increase a risk of recurrence or persistence of DME after STTA injection.

The results of two large cohort studies indicated that glitazone is a risk factor for developing DME [21, 22], and we have reported the first case of DME after pioglitazone use in Japan [23]. However, in this study, pioglitazone was not found to be a risk factor for recurrence or persistence of DME after a STTA injection.

From this study, we tentatively suggest the indication of STTA in the clinical practice. STTA may be selected for treatment of DME without MAs or DME after vitrectomy. DME with MAs may be treated with intravitreal injection of anti-VEGF antibodies. SMDLP may not be fitted with STTA. Further studies are needed to evaluate the additive effect of 
intravitreal injection of anti-VEGF antibodies with SMDLP for patients with DME.

\section{Conclusions}

Although this study has a limitation because of its retrospective nature, patients who needed to have combined MAPC and SMDLP with a STTA injection had significantly higher refractoriness to DME. However, vitrectomized eyes may reduce the incidence of recurrence or persistent DME after a STTA injection. Additional prospective studies are needed to confirm the risk factors for a recurrence or persistent DME after a STTA injection.

\section{Conflict of Interests}

The authors declare that there is no conflict of interests regarding the publication of this paper.

\section{Acknowledgments}

This study was supported by a grant from The Eye Research Foundation for the Aged and Charitable Trust Fund for Ophthalmic Research in Commemoration of Santen Pharmaceutical's Founder and a Grant-in-Aid from the Ministry of Education, Science, Sports, and Culture of the Japanese Government. The authors thank Professor Duco Hamasaki for editing the paper.

\section{References}

[1] M. O’Doherty, I. Dooley, and M. Hickey-Dwyer, "Interventions for diabetic macular oedema: a systematic review of the literature," British Journal of Ophthalmology, vol. 92, no. 12, pp. 15811590, 2008.

[2] J. W. Y. Yau, S. L. Rogers, R. Kawasaki et al., "Global prevalence and major risk factors of diabetic retinopathy," Diabetes Care, vol. 35, no. 3, pp. 556-564, 2012.

[3] P. Mitchell, F. Bandello, U. Schmidt-Erfurth et al., "The RESTORE study: ranibizumab monotherapy or combined with laser versus laser monotherapy for diabetic macular edema," Ophthalmology, vol. 118, no. 4, pp. 615-625, 2011.

[4] M. J. Elman, H. Qin, and L. P. Aiello, "Intravitreal ranibizumab for diabetic macular edema with prompt versus deferred laser treatment: three-year randomized trial results," Ophthalmology, vol. 119, no. 11, pp. 2312-2318, 2012.

[5] M. B. Sultan, D. Zhou, J. Loftus, T. Dombi, and K. S. Ice, "A phase 2/3, multicenter, randomized, double-masked, 2-year trial of pegaptanib sodium for the treatment of diabetic macular edema," Ophthalmology, vol. 118, no. 6, pp. 1107-1118, 2011.

[6] D. V. Do, Q. D. Nguyen, D. Boyer et al., "One-year outcomes of the da VINCI study of VEGF trap-eye in eyes with diabetic macular edema," Ophthalmology, vol. 119, no. 8, pp. 1658-1665, 2012.

[7] T. S. Kern, "Contributions of inflammatory processes to the development of the early stages of diabetic retinopathy," Experimental Diabetes Research, vol. 2007, Article ID 95103, 14 pages, 2007.

[8] E. A. Felinski and D. A. Antonetti, "Glucocorticoid regulation of endothelial cell tight junction gene expression: novel treatments for diabetic retinopathy," Current Eye Research, vol. 30, no. 11, pp. 949-957, 2005.

[9] M. Nauck, M. Roth, M. Tamm et al., "Induction of vascular endothelial growth factor by platelet-activating factor and platelet-derived growth factor is downregulated by corticosteroids," American Journal of Respiratory Cell and Molecular Biology, vol. 16, no. 4, pp. 398-406, 1997.

[10] J. E. Sears and G. Hoppe, “Triamcinolone acetonide destabilizes VEGF mRNA in Müller cells under continuous cobalt stimulation," Investigative Ophthalmology \& Visual Science, vol. 46, no. 11, pp. 4336-4341, 2005.

[11] N. Ohguro, A. A. Okada, and Y. Tano, "Trans-Tenon's retrobulbar triamcinolone infusion for diffuse diabetic macular edema," Graefe's Archive for Clinical and Experimental Ophthalmology, vol. 242, no. 5, pp. 444-445, 2004.

[12] T. C. Ho and W. W. Lai, "Intravitreal cortisone injection for refractory diffuse diabetic macular edema," Ophthalmologica, vol. 220, no. 5, pp. 349-350, 2006.

[13] T. Sakamoto, T. Hida, Y. Tano et al., "Committee for Triamcinolone Acetonide for Ocular Disease in Japan: survey of triamcinolone acetonide for ocular diseases in Japan," Nihon Ganka Gakkai Zasshi, vol. 111, no. 12, pp. 936-945, 2007.

[14] S. Nonomura, T. Oshitari, M. Arai et al., "The effect of posterior sub-Tenon's capsule triamcinolone acetonide injection to that of pars plana vitrectomy for diabetic macular edema," Clinical Ophthalmology, vol. 8, pp. 825-830, 2014.

[15] H.-P. Qi, S. Bi, S.-Q. Wei, H. Cui, and J.-B. Zhao, "Intravitreal versus subtenon triamcinolone acetonide injection for diabetic macular edema: a systematic review and meta-analysis," Current Eye Research, vol. 37, no. 12, pp. 1136-1147, 2012.

[16] Y. Takatsuna, S. Yamamoto, Y. Nakamura, T. Tatsumi, M. Arai, and Y. Mitamura, "Long-term therapeutic efficacy of the subthreshold micropulse diode laser photocoagulation for diabetic macular edema," Japanese Journal of Ophthalmology, vol. 55, no. 4, pp. 365-369, 2011.

[17] M. L. Ribeiro, S. G. Nunes, and J. G. Cunha-Vaz, "Microaneurysm turnover at the macula predicts risk of development of clinically significant macular edema in persons with mild nonproliferative diabetic retinopathy," Diabetes Care, vol. 36, no. 5, pp. 1254-1259, 2013.

[18] C. Haritoglou, M. Kernt, A. Neubauer et al., "Microaneurysm formation rate as a predictive marker for progression to clinically significant macular edema in nonproliferative diabetic retinopathy," Retina, vol. 34, no. 1, pp. 157-164, 2014.

[19] R. Klein, B. E. K. Klein, S. E. Moss, and K. J. Cruickshanks, "The Wisconsin epidemiologic study of diabetic retinopathy. XV. The long-term incidence of macular edema," Ophthalmology, vol. 102, no. 1, pp. 7-16, 1995.

[20] T.-H. Chou, P.-C. Wu, J. Z.-C. Kuo, C.-H. Lai, and C.-N. Kuo, "Relationship of diabetic macular oedema with glycosylated haemoglobin," Eye, vol. 23, no. 6, pp. 1360-1363, 2009.

[21] D. S. Fong and R. Contreras, "Glitazone use associated with diabetic macular edema," American Journal of Ophthalmology, vol. 147, no. 4, pp. 583.e1-586.el, 2009.

[22] I. Idris, G. Warren, and R. Donnelly, "Association between thiazolidinedione treatment and risk of macular edema among patients with type 2 diabetes," Archives of Internal Medicine, vol. 172, no. 13, pp. 1005-1011, 2012.

[23] T. Oshitari, N. Asaumi, M. Watanabe, K. Kumagai, and Y. Mitamura, "Severe macular edema induced by pioglitazone in a patient with diabetic retinopathy: a case study," Vascular Health and Risk Management, vol. 4, no. 5, pp. 1137-1140, 2008. 


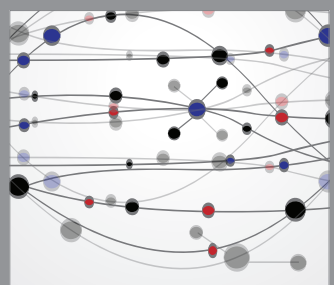

The Scientific World Journal
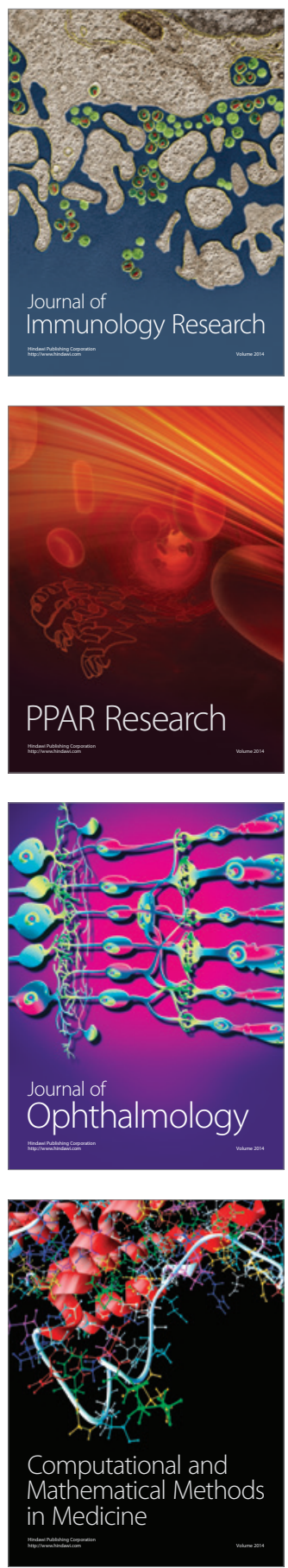

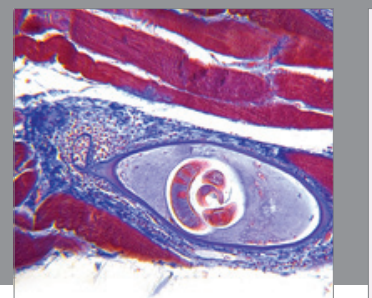

Gastroenterology

Research and Practice
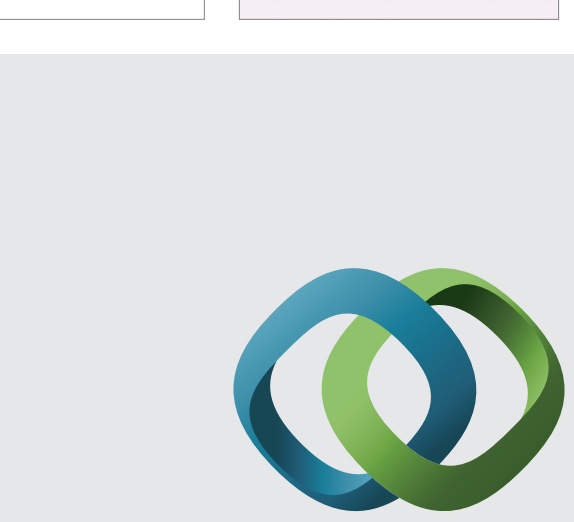

\section{Hindawi}

Submit your manuscripts at

http://www.hindawi.com
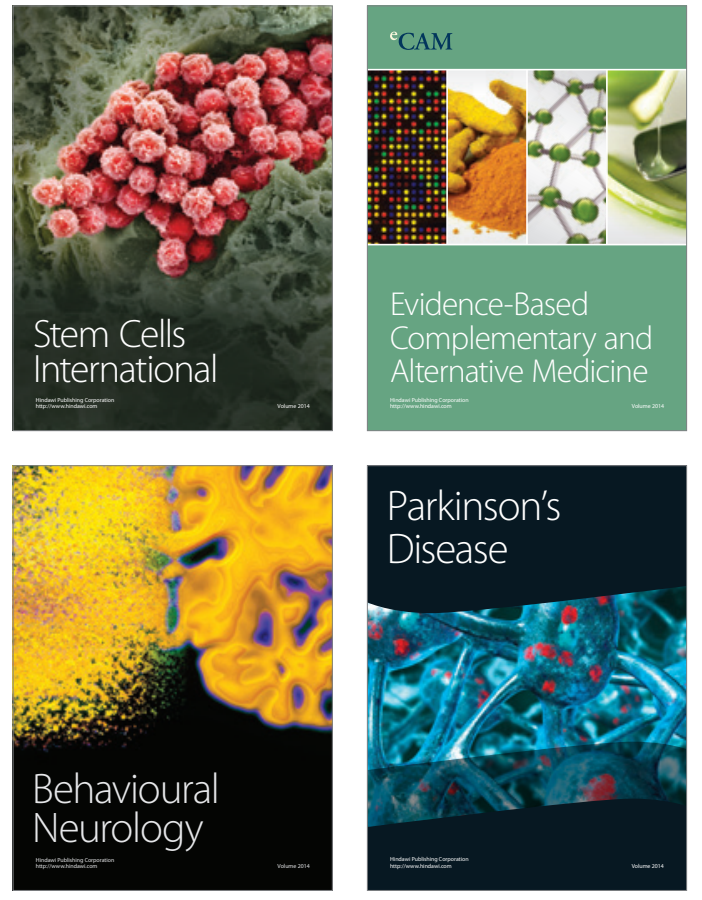
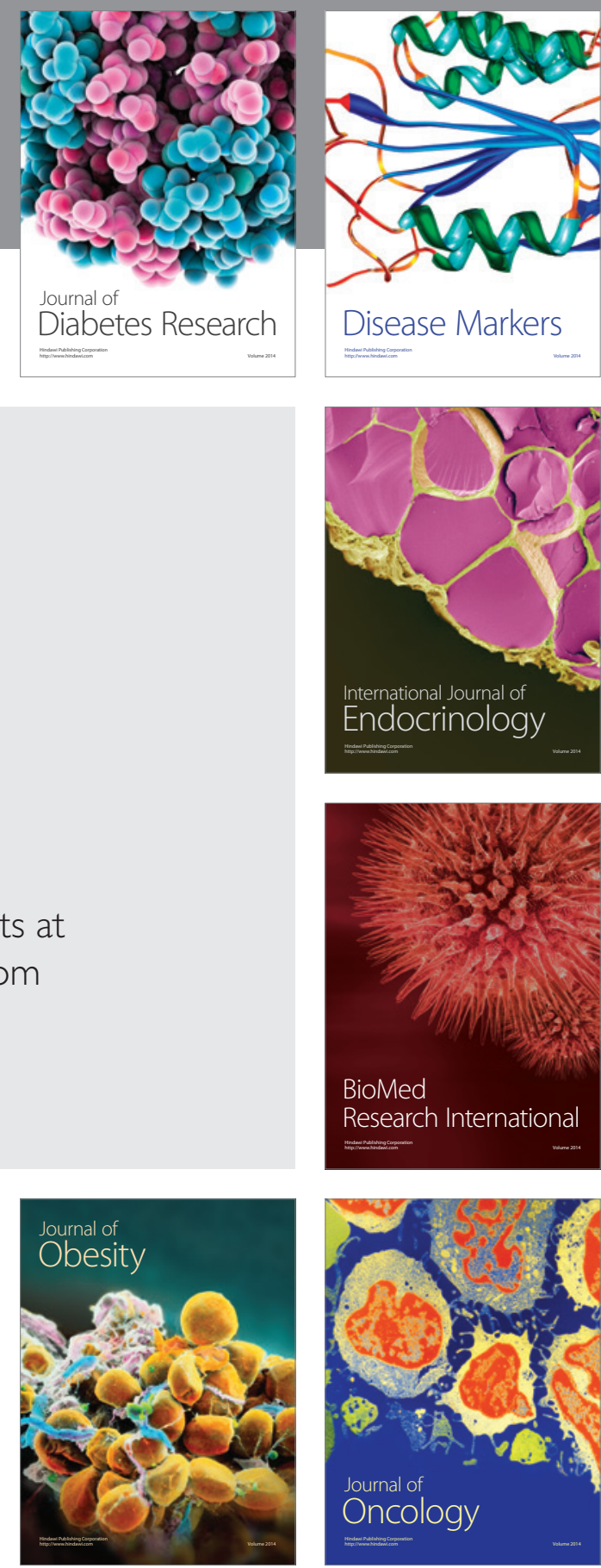

Disease Markers
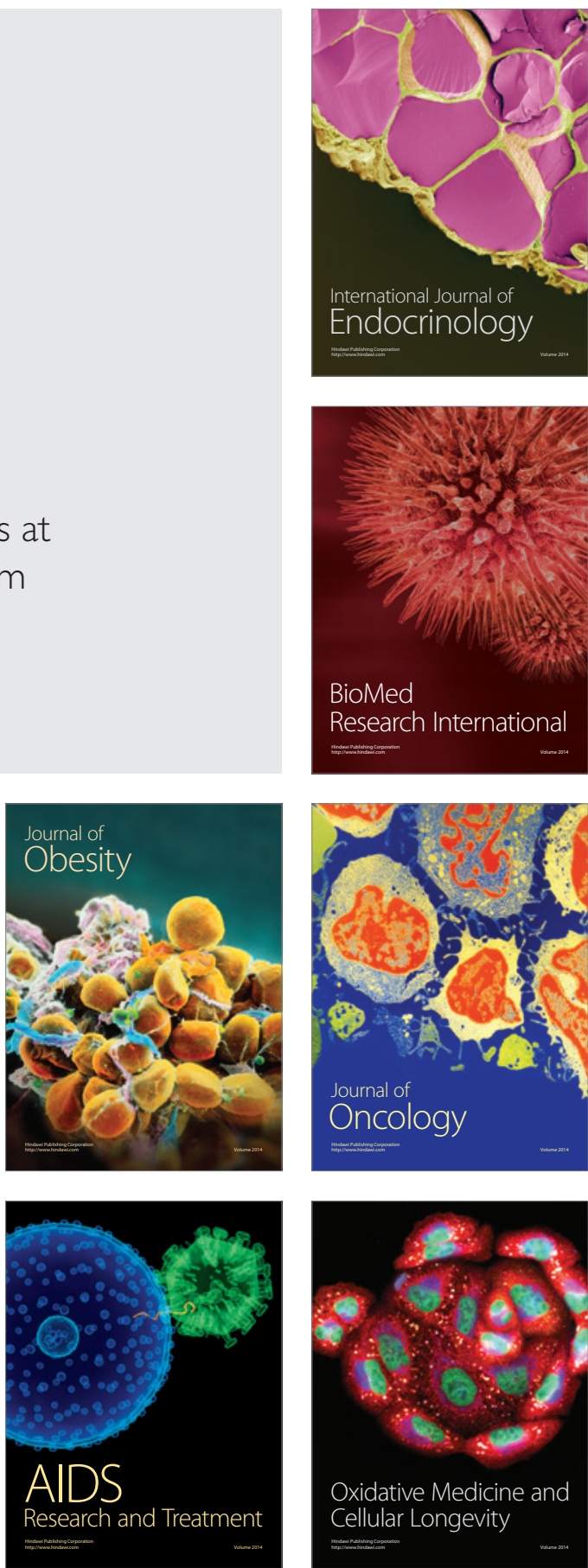